\title{
Software Engineering Development and Analysis of Life Cycle Models
}

\author{
Mubarak Elamin Elmubarak Daleel \\ University of Jeddah (Kingdom of Saudi Arabia) \\ Faculty of Computer \& Information Technology Department of Information System \\ AlzaeimAlazhari University (Sudan) \\ Faculty of Computer science \& Information Technology Department of computer science
}

\begin{abstract}
We will address in this search for an important issue in the field of the computer world, which has grown a tremendous way in the late twentieth century and become the defining feature more diverse and complicated at the same time, known as the software development life cycle which is about five models they waterfall model and Incrementalmodel-shaped $\mathrm{v}$ model Spiral and RAD model and we will address the advantages and disadvantages of each model, the main objective of this research is the representation of the different models of software development work and compare
\end{abstract}

\section{Keywords}

Software Development Process, analysis of model life cycle, Comparison between five models of Software Engineering.

\section{INTRODUCTION}

With the development of computer use increased development and complexity of information technology has emerged a huge number of applications to software engineering that help the transactions included and are linking information and data business office also requires harnessing technology for the user to create a system that meets its purpose and that the development of software in order to use the framework to be planning, management and control of the process the development of the information system. [1] Software engineering is the application of systems or systematic approach measurable quantitative for the development, operation and maintenance of software systems .

The software development process is a software product development and there are several models for such processes This process is designed to determine the criteria required for the development and maintenance of software. [2]

There are five phases in every Software development life cycle model :

\section{REQUIREMENT GATHERING AND ANALYSIS}

At this stage it is grouped business requirements and this stage is the main focus of project managers and company owners are meeting with managers and owners of companies to identify needs such as determining how to use the system and what data must be input into the system process and what data outputs are in this system process are the questions required to answer them at this stage

\subsection{Design}

At this stage it is to prepare the system and software design helps to identify the hardware and system requirements and also helps determine the overall system architecture and system design specifications serve as input for the next stage

\subsection{Implementation or coding}

At this stage are receiving system design and documentation work is divided into units and the start of actual coding and also at this stage is the production of code for each give rise for the main focus of the process of development and this stage is the longest stages of the software development

\subsection{Testing}

At this stage is to choose the icon in the light of the requirements to ensure that the product is used to solve the needs of the development phase of the software are also at this stage the unit testing and integration testing and acceptance testing and system testing to make sure the face of the requirements phase

\subsection{Deployment}

At this stage the product is delivered to the customer and published for use in the market after the successful experiences

\subsection{Maintenance}

At this stage is taken care of advanced product is also known as the maintenance of that when customers start using the development system comes from actual problems and need to be resolved from time to time.

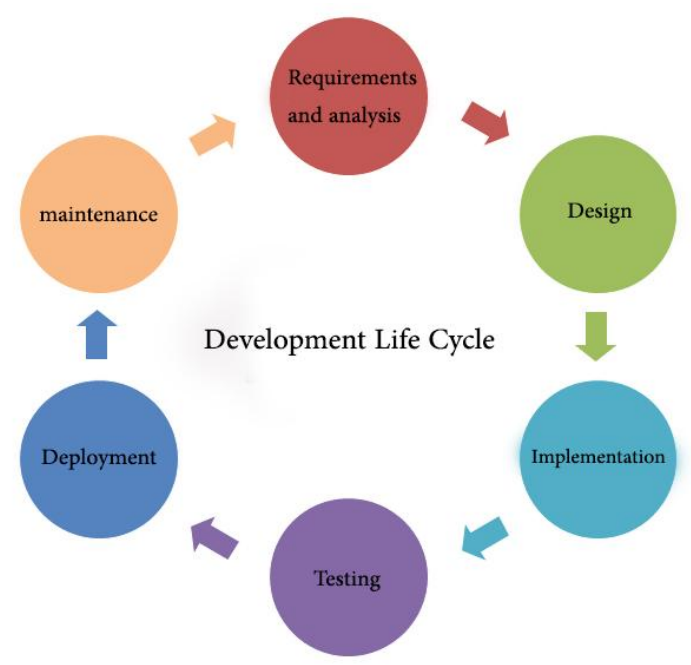

Figure ( 1 ) : software Development Life Cycle

\section{SOFTWARE DEVELOPMENT LIFE CYCLE MODELS}

3.1 Waterfall Model 
Will speak here about the waterfall model is a classical model for software engineering and is considered the model is the oldest development models and is used widely in government projects and a lot of big companies and also being planned for this model in the early stages it ensures design flaws before the development process and in addition to the so he and document intensive make it work well for projects in which the product quality strict control be.It consists waterfall model life cycle of several stages of non-overlapping start with determining the system requirements and software requirements and continue with the architectural design and detailed coding, testing and maintenance, and is also used waterfall model for many other life-cycle model.[3]

\subsubsection{Basic Principles}

1. This project is divided into sequential stages with some overlap between the stages.

2. The focus is on planning and arranging schedules and deadlines as well as the objectives and budgets and implement it in the entire system at one time

3. The tight control over the life of the project through written documentation and approval by the user and administrative information technology which are made in the end of the stage before the start of the next phase signature

\subsubsection{Advantages}

Featuring model waterfall is that it enables empowerment and control was to set a timetable with deadlines for each stage of product development and can move through the stages and the model development and one after another through the design, implementation, testing, installation and problems and ends in the operation and maintenance

\subsubsection{Disadvantages}

Disadvantages of waterfall model is that it does not allow for a lot of meditation or review as an example in the testing phase, it is very difficult to go back and change anything that has not been well documented.

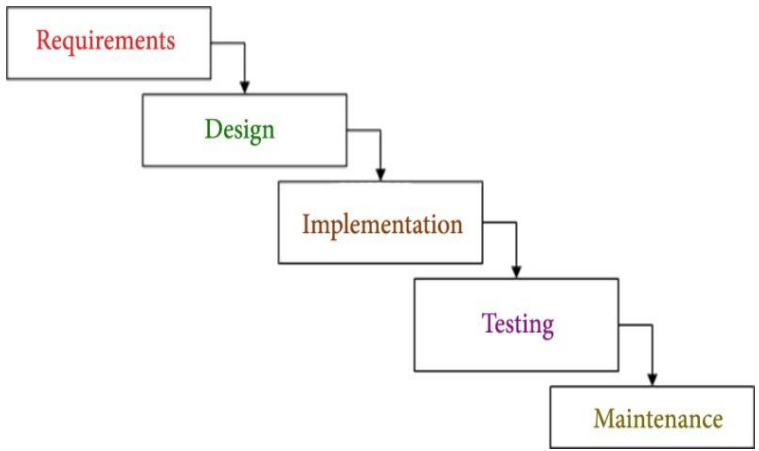

Fig ( 2 ) Original Waterfall Model

\subsection{Incremental model}

Here we will talk about the progressive model is an approach to the waterfall model, and here the development of multiple sessions taking place which makes the life cycle (multiple Waterfall) is divided into sessions so as to reach younger and be duplicates much easier and all the duplicates pass through the phases of requirements, design, implementation and testing session

This is the production version of the software operates through the first iteration so that you have a program of work at an early date through the software life cycle and subsequent iterations based on the first program.

\subsubsection{Basic Principle}

1. This model handles a new way to develop systems that can be faster results and requires less information and provide greater flexibility

2. The project is divided into small parts here and will allow the team the possibility of development to show the results in early and get valuable information by users of the system

3. Here most of the repetition is actually a miniwaterfall in the process stage to provide information for the design of the next phase of software development.

\subsubsection{Advantages}

1. is the best software process models

2. allows reactions to the phases of the system

3. can be used so as not well understood requirements

\subsubsection{Disadvantages}

1. It is not easy to control in this model

2. and does not use clear milestones in the development process

3. Do not be finished stage

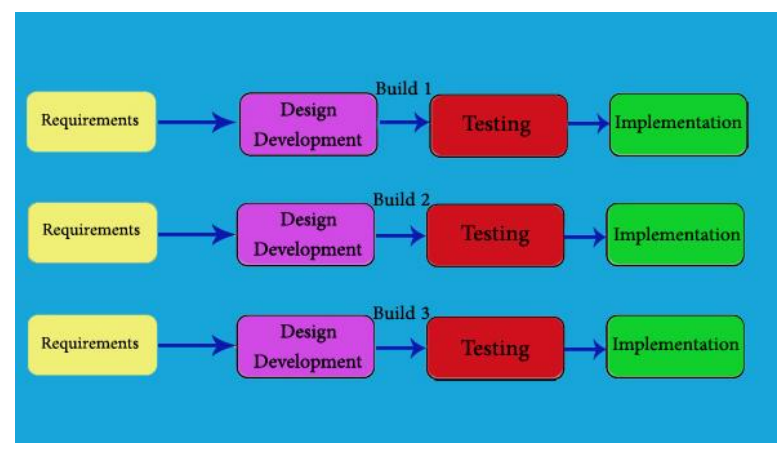

Fig ( 3 ) Incremental life cycle model

\section{3 shaped Model}

$\mathrm{V}$-Shaped life cycle is a sequential path of the implementation of the operations at this stage must be completed each stage before they begin the next phase in this model is the development of testing procedures in the life cycle in advance before you make any encoding is implementation of each phase of during the previous stages and requirements of life model just like in the waterfall model and here before the start of the development is the establishment of the system test plan and the plan focused on meeting the requirements of jobs in the collection limited to software development process.

\subsubsection{Advantages}

1. This model is simple and easy to use

2. at every stage there is a specific output

3. chance of success in this largest of the waterfall model because of the development of test plans during the life cycle model

4. works well for small enterprises so as to understand the requirements easily 


\subsubsection{Disadvantages}

1. Example strict waterfall model

2. a little flexibility and scope of the difficult and costly adjustment model

3. Here are software development through the implementation phase so it is not an early production models

4. there is no clear path to the problems that are found in the testing phase [4].

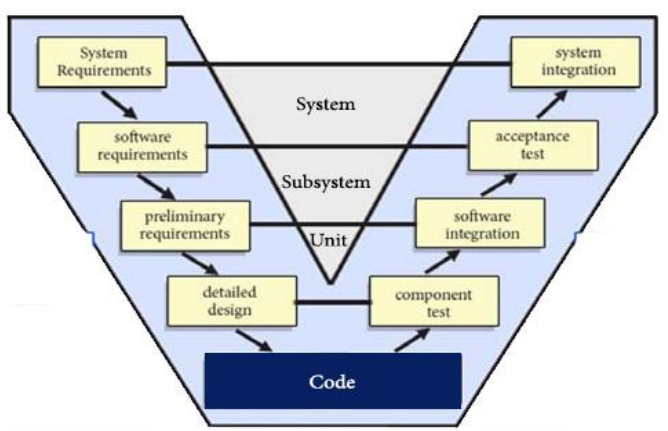

Fig.( 4 ) V shaped Model

\subsection{Spiral Model}

Here we will talk about the spiral model is similar to the model of the progressive model with the possibility of risk analysis and consists of a cycle of four phases: planning model and risk analysis and evaluation repeatedly passing model through these stages in redundancy and called spirals in this model starts from planning and assembly requirements phase and evaluation phase risks and built all the basic spiral

\subsubsection{Basic Principles}

1. The focus is on risk assessment, as well as minimize them through the project is divided into smaller parts and provide more ease of change during the development process and by providing the opportunity to assess the risks and provide security throughout the life cycle

2. each session where the evolution during the same sequence of steps for each part of the product and all levels of preference among the practical and down to encode each program separately

3. spiral model the four main parts are setting goals and alternatives and imposed restrictions on the redundancy process and secondly evaluate alternatives and thirdly identify and resolve risks and fourthly the development and verification of completion

4. start of each session to select and identify the owners of companies and the conditions of the process of winning and ending each session with the participation and commitment

\subsubsection{Advantages}

1. many risks

2. be good for large projects with a very critical tasks

3. is the production of programs in the software life cycle very early

\subsubsection{Disadvantages}

1. be charged for the use of model

2. requires very specific risk analysis experience

3. success of the project depends on the risk analysis stage

4. do not work well for small projects

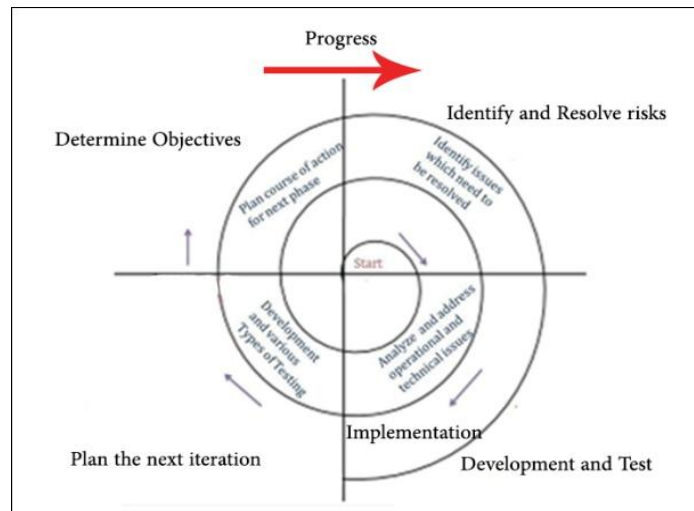

Fig. ( 4 ) Spiral Model

\subsection{RAD Model}

Rapid Application Development (RAD) model is a model of the additional software that is based on the development in the short life cycle process and is considered the model is a highspeed model that adapts to many of the steps with a waterfall that achieves rapid development using based approach to a form here and in the case of whether the project requirements and scope of the known project well then RAD process then enables the product development team full energy to create any software system in a very short period of time be in a few days and RAD model is like the other models in the operations of joint activities and tire Home.

RAD model in the planning of the program there are several teams working simultaneously on various system functions and includes three main stages

\section{Business modeling \\ 2. Data modeling \\ 3. Process modeling}

Construction focuses mainly on the use of existing software components and the application of automatic code generation. In the last stage Deployment establishes a basis for iterations

\subsubsection{Advantages}

1 be flexible and adaptable to changes

2 applications gives users a description from which to rule whether the system requirements are met by the system and report the results can be compared with the current report can be reviewed data entry forms and data access

3 RAD includes a short development cycles and see the users of the product quickly

4 RAD involves the participation of users and thus increase the chances of acceptance at an early date

$5 \mathrm{RAD}$ there is a reduction in project risk

6 be results in the reduction of the costs of setting up the system

\subsubsection{Disadvantages}


1 the cost of non-specific products can be mitigated by the client by agreeing to a limited amount of rewriting RAD

2 it can be difficult commitment of time to the success of RAD process.
The comparison between different models is shown by their advantages and disadvantages in table

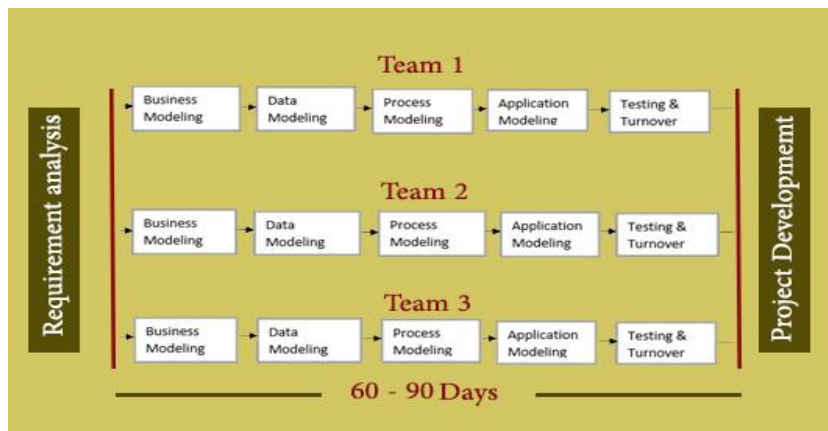

Fig.( 5 ) - RAD Model

Table1 Comparison of five models

\begin{tabular}{|c|c|c|c|c|c|}
\hline Features & Waterfall & Incremental & V shaped & Spiral & RAD \\
\hline $\begin{array}{c}\text { Requirement } \\
\text { specifications }\end{array}$ & Beginning & Beginning & Beginning & Beginning & $\begin{array}{c}\text { Time boxed } \\
\text { release }\end{array}$ \\
\hline $\begin{array}{c}\text { Understanding } \\
\text { Requirement }\end{array}$ & Well understood & NotWell understood & Well understood & Well understood & $\begin{array}{c}\text { NotWell } \\
\text { understood }\end{array}$ \\
\hline Cost & Low & Low & Expensive & Expensive & Low \\
\hline Simplicity & Simple & Intermediate & Simple & Intermediate & Very simple \\
\hline $\begin{array}{c}\text { Risk Analysis } \\
\text { Flexibility to } \\
\text { change }\end{array}$ & High & Easily manageable & Low & Low & Easy \\
\hline $\begin{array}{c}\text { User Involvement } \\
\text { Only at beginning }\end{array}$ & Intermediate & High & High & Only at the \\
beginning
\end{tabular}

\section{CONCLUSION}

In this study, we compared between different software development life cycle model based on common factors among them, as well as risks that correspond to each other and on the basis of the cost of these features for a particular project and one can decide and he has to be and choose the program to model the life of software development in particular cycle. The right to choose between them because of the life-cycle model is very important in the software industry must take into account the time limit and be of the required quality and within the estimated costs

This study will make the process of selecting the SDLC model Iterative water fall model overcome the drawback of original waterfall model It allow feedback to proceeding stage. Prototype model used to develop online systems for transaction processing. Since significantly reduce rework and lead to the creation of working model in lower capital cost. Spiral model is used for development of large, complicated and expensive projects like scientific Projects .Since spiral model approach enables the project term to address the highest risk at the lowest total cost

\section{REFERENCES}

[1] IanSommerville,SoftwareEngineering,AddisonWesley,9t $\mathrm{h}$ ed., 2010

[2] Comparative Analysis of Different types of Models in SoftwareDevelopment Life CycleMs.ShikhaMaheshwari, Prof. Dinesh Ch.Jain

[3] A Comparison between Five Models of Software Engineering NabilMohammed Ali Munassar and A.Govardhan 
[4] National Instruments Corporation, "Lifecycle Models",2006 ,http://zone.ni.com.

[5] Gray P ilgrim, "Waterfall Model, Website http://www.buzzle.com/articles/waterfall model.html, Jan, 2012

[6] Dr.DeepshikhaJamwal"Analysis of software Development Models", ISSN: 2229 4333(print),ISSN:09768491(online), vol.1ISSUE 2, December 2010.
[7] SwapanjaIngale "Comparative Study Of Software Development Model" International conference on advances in computing \&management 2012

[8] Maglyas, A.; Nikula, U.; Smolander, K.,"Comparison of two models of success prediction in software development projects", Software Engineering Conference (CEE-SECR), 2010 6th Central and Eastern European on 13-15 Oct. 2010, pp. 43-49.

[9] Sanjana Taya "Comparative Analysis of Software Development Life" ISSN:2229-4333(print),ISSN:09768491(online),vol.2ISSUE 4,Oct-Dec2011 